\title{
Manipulating Topological Domain Boundaries in the Single-Layer Quantum Spin Hall Insulator $1 \mathrm{~T}^{\prime}-\mathrm{WSe}_{2}$
}

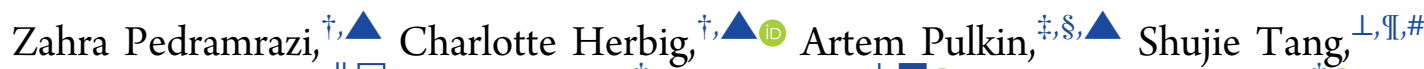

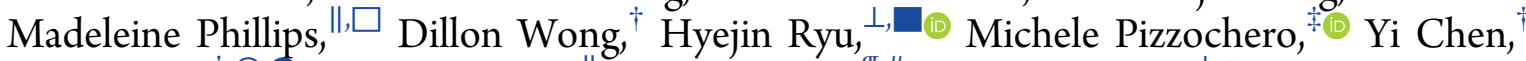

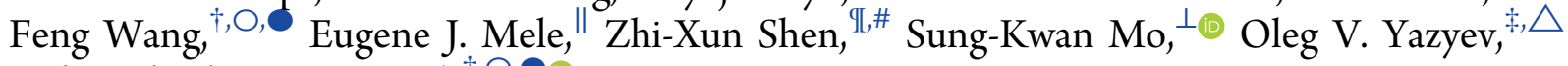
and Michael F. Crommie ${ }^{*, \dagger, O,}$

${ }^{\dagger}$ Department of Physics, University of California at Berkeley, Berkeley, California 94720, United States

${ }^{\ddagger}$ Institute of Physics, Ecole Polytechnique Fédérale de Lausanne (EPFL), CH-1015 Lausanne, Switzerland

${ }^{\S}$ Division of Chemistry and Chemical Engineering, California Institute of Technology, Pasadena, California 91125, United States

${ }^{\perp}$ Advanced Light Source, Lawrence Berkeley National Laboratory, Berkeley, California 94720, United States

${ }^{\text {II }}$ Stanford Institute for Materials and Energy Sciences, SLAC National Accelerator Laboratory, Menlo Park, California 94025, United States

${ }^{\#}$ Geballe Laboratory for Advanced Materials, Departments of Physics and Applied Physics, Stanford University, Stanford, California 94305, United States

"Department of Physics and Astronomy, University of Pennsylvania, Philadelphia, Pennsylvania 19104, United States

$\square$ Center for Computational Materials Science, Naval Research Laboratory, Washington, D.C. 20375, United States

- Center for Spintronics, Korea Institute of Science and Technology, Seoul 02792, Korea

${ }^{\circ}$ Materials Sciences Division, Lawrence Berkeley National Laboratory, Berkeley, California 94720, United States

- Kavli Energy NanoScience Institute at the University of California Berkeley and the Lawrence Berkeley National Laboratory, Berkeley, California 94720, United States

$\triangle$ National Centre for Computational Design and Discovery of Novel Materials MARVEL, Ecole Polytechnique Fédérale de Lausanne (EPFL), CH-1015 Lausanne, Switzerland

Supporting Information

ABSTRACT: We report the creation and manipulation of structural phase boundaries in the single-layer quantum spin Hall insulator $1 \mathrm{~T}^{\prime}-\mathrm{WSe}_{2}$ by means of scanning tunneling microscope tip pulses. We observe the formation of onedimensional interfaces between topologically nontrivial $1 \mathrm{~T}^{\prime}$ domains having different rotational orientations, as well as induced interfaces between topologically nontrivial $1 \mathrm{~T}^{\prime}$ and topologically trivial $1 \mathrm{H}$ phases. Scanning tunneling spectroscopy measurements show that $1 \mathrm{~T}^{\prime} / 1 \mathrm{~T}^{\prime}$ interface states are localized at domain boundaries, consistent with theoretically predicted unprotected interface modes that form dispersive bands in and around the energy gap of this quantum spin Hall insulator. We observe a qualitative difference in the experimental spectral line shape between topologically "unprotected" states at $1 \mathrm{~T}^{\prime} / 1 \mathrm{~T}^{\prime}$ domain boundaries and protected states at $1 \mathrm{~T}^{\prime} / 1 \mathrm{H}$ and $1 \mathrm{~T}^{\prime} /$ vacuum boundaries in single-layer $\mathrm{WSe}_{2}$.

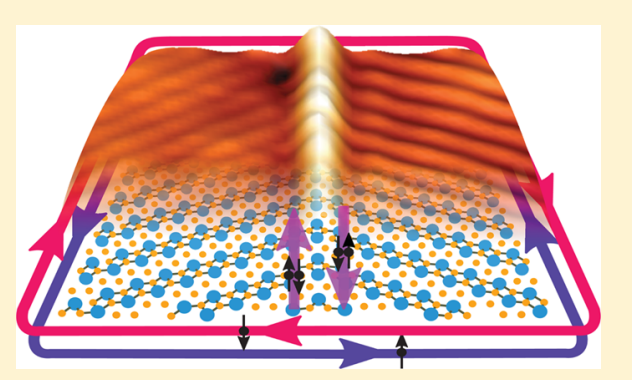

KEYWORDS: Scanning tunneling microscopy, transition metal dichalcogenides, quantum spin hall insulators, domain boundary, ferroelasticity

$\mathrm{R}$ ecent experimental studies have reported the observation of the quantum spin Hall (QSH) effect in single layers of the transition metal dichalcogenides (TMDs) $\mathrm{WTe}_{2}$ and $\mathrm{WSe}_{2}$ in the $1 \mathrm{~T}^{\prime}$ structural phase. ${ }^{1-5}$ Evidence of the QSH state includes inverted bandgaps, ${ }^{1}$ topologically protected edge states, ${ }^{1,2,5}$ and quantized edge conduction of $\mathrm{e}^{2} / \mathrm{h}$ per edge. ${ }^{3,4}$ QSH edge states have been observed to reside at $1 \mathrm{~T}^{\prime} / 1 \mathrm{H}$ and $1 \mathrm{~T}^{\prime} /$ vacuum boundaries, both of which are interfaces between nontrivial $\left(1 \mathrm{~T}^{\prime}-\mathrm{TMD}\right)$ and trivial $(1 \mathrm{H}-$
TMD phase or vacuum) media. ${ }^{1,2,5}$ Such interfaces are expected to host topologically protected edge states. ${ }^{6-11} \mathrm{~A}$ less well-studied type of boundary in quantum spin Hall insulator (QSHI) materials is the interface between different nontrivial domains where the $\mathrm{Z}_{2}$ topological invariant of the

Received: May 27, 2019

Revised: July 6, 2019

Published: July 22, 2019 
a

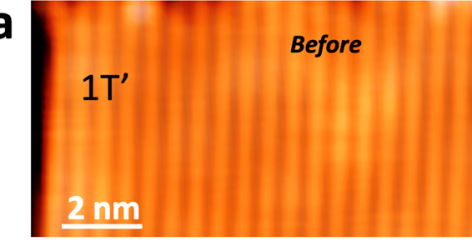

C

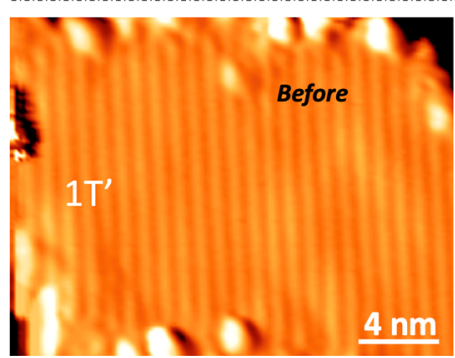

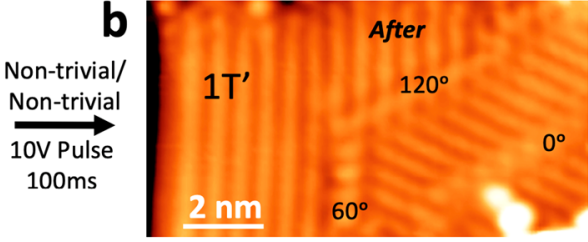

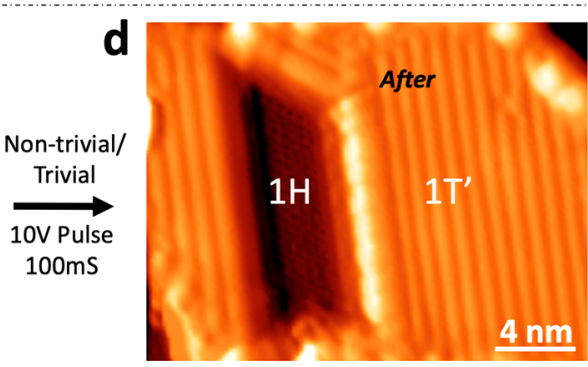

Figure 1. STM tip-induced structural change in monolayer $1 \mathrm{~T}^{\prime}-\mathrm{WSe}_{2}$. STM topographic images of a monolayer $1 \mathrm{~T}^{\prime}-\mathrm{WSe}_{2}$ island (a) before and (b) after applying a tip voltage pulse $\left(V_{\text {pulse }}=10 \mathrm{~V}, \Delta t=100 \mathrm{~ms}\right.$, tip-surface separation $\left.=6 \AA\right)$. The tip pulse creates $1 \mathrm{~T}^{\prime} / 1 \mathrm{~T}^{\prime}$ domain boundaries having different rotational orientations. STM topographic images show a different island (c) before and (d) after an applied tip voltage pulse $\left(V_{\text {pulse }}=10 \mathrm{~V}, \Delta t=100 \mathrm{~ms}\right.$, tip-surface separation $\left.=6 \AA\right)$ induces a $1 \mathrm{~T}^{\prime}$ to $1 \mathrm{H}$ structural phase transition near the center of the island. $V_{s}=1 \mathrm{~V}, I_{\mathrm{t}}=$ $10 \mathrm{pA}, T=4.5 \mathrm{~K}$ for all images. (Image intensity here is proportional to $\mathrm{d} z / \mathrm{d} x$ (where $z$ is height) to enhance contrast between regions having different structural phases.)
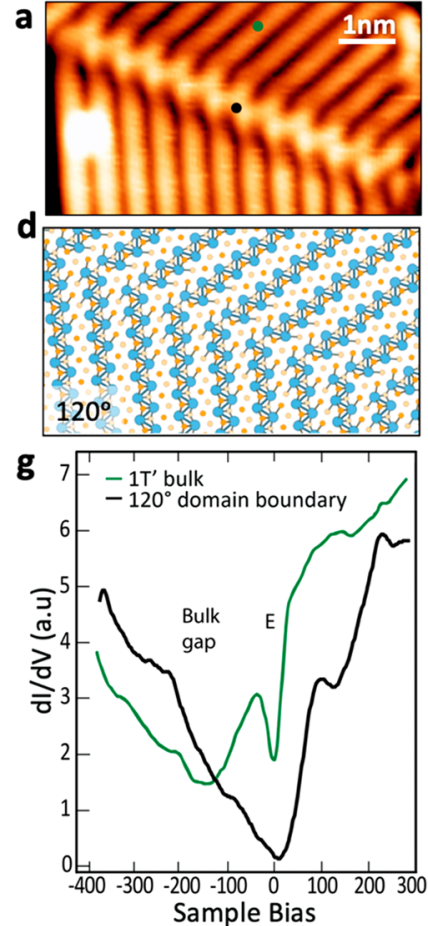
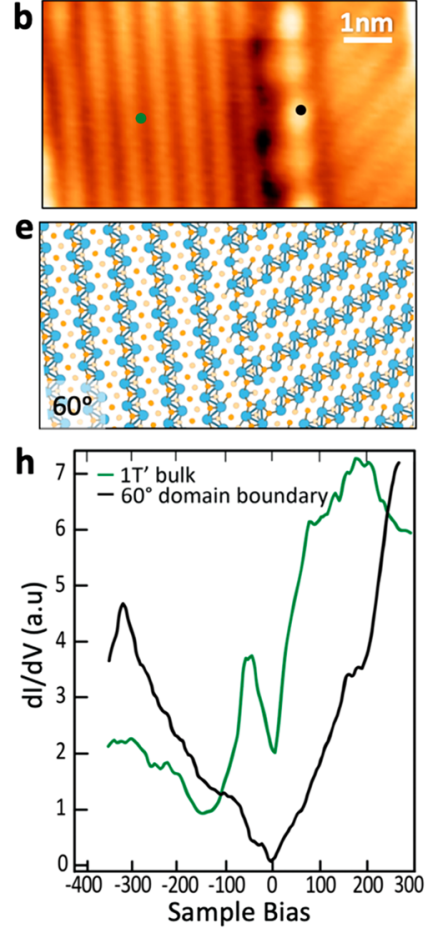
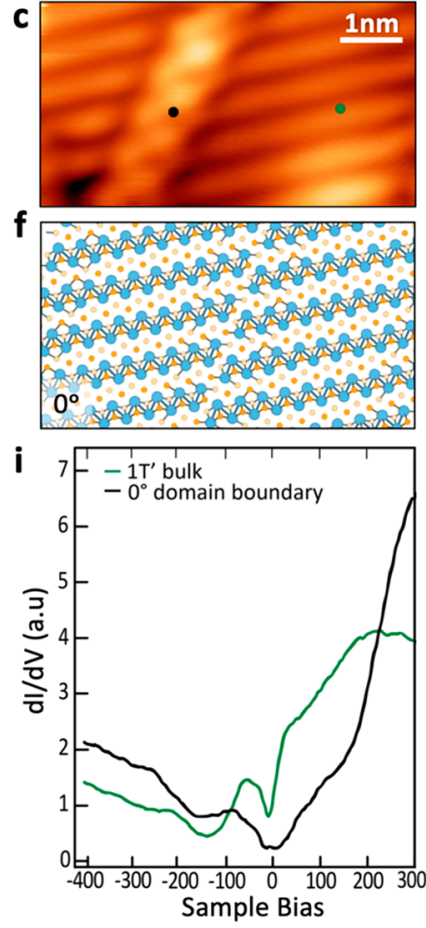

Figure 2. Structural and electronic properties of $1 \mathrm{~T}^{\prime} / 1 \mathrm{~T}^{\prime}$ domain boundaries. STM images of (a) $120^{\circ}$, (b) $60^{\circ}$, and (c) $0^{\circ} 1 \mathrm{~T}^{\prime} / 1 \mathrm{~T}^{\prime}$ domain boundaries in $1 \mathrm{~T}^{\prime}-\mathrm{WSe}_{2}\left(V_{\mathrm{s}}=1 \mathrm{~V}, I_{\mathrm{t}}=10 \mathrm{pA}\right.$, standard STM topographs). Relaxed structural models of (d) $120^{\circ}$, (e) $60^{\circ}$, and (f) $0^{\circ}$ domain boundaries in $1 \mathrm{~T}^{\prime}-\mathrm{WSe}_{2}$ (calculated using DFT). STM dI/dV spectroscopy measured at $(\mathrm{g}) 120^{\circ}$, (h) $60^{\circ}$, and (i) $0^{\circ}$ domain boundaries compared to the bulk for single-layer $1 \mathrm{~T}^{\prime}-\mathrm{WSe}_{2}$ (spectroscopy positions marked by black and green dots in panels a-c $)\left(f=613.7 \mathrm{~Hz}, V_{\mathrm{ac}}=4 \mathrm{mV}\right.$ $T=4.5 \mathrm{~K}$; initial tunneling parameters for spectroscopy measurements: $V_{\mathrm{s}}=-400 \mathrm{mV}, I=100 \mathrm{pA}$ ).

bulk does not change across the interface. We refer to a domain boundary as "topological" when there is a change in the topological invariant across the interface and "trivial" when the invariant is the same on either side of the domain wall. A recent theoretical study of charge transport in quantum Hall insulators with trivial interfaces predicted that conduction through otherwise dissipationless quantum Hall edge states can be controllably deflected into trivial interface states, thus enabling gate-tunable charge and spin transport. ${ }^{12}$ Single-layer
TMD materials provide a new strategy for constructing such coexisting topological and trivial interfaces by switching the layer structural phase via some local stimuli. ${ }^{13-17}$ For example, the $1 \mathrm{~T}^{\prime}$ phase has recently been predicted to be ferroelastic in single layers, which suggests that it can be switched between different dimerization orientations by applied stress. This provides a mechanism to induce topologically trivial interfaces between QSHI domains with different crystallographic orientations. A similar strategy could allow generation of 
topologically nontrivial interfaces by inducing local phase switching between structures that have different $Z_{2}$ indices. ${ }^{18,19}$

Here we report the local phase manipulation of single-layer $1 \mathrm{~T}^{\prime}-\mathrm{WSe}_{2}$ for the purpose of creating two kinds of onedimensional interfaces: (1) trivial interfaces between two $1 \mathrm{~T}^{\prime}$ domains and (2) topological interfaces between $1 \mathrm{~T}^{\prime}$ and $1 \mathrm{H}$ domains. By using scanning tunneling microscope (STM) tip pulses, we are able to locally switch from the $1 \mathrm{~T}^{\prime}$ phase to the $1 \mathrm{H}$ phase of $\mathrm{WSe}_{2}$, as well as between different orientations of the $1 \mathrm{~T}^{\prime}$ phase. $1 \mathrm{~T}^{\prime} / 1 \mathrm{~T}^{\prime}$ domain-boundary formation is observed to be reversible, which supports the conjecture that single-layer $1 \mathrm{~T}^{\prime}-\mathrm{WSe}_{2}$ is a ferroelastic material. ${ }^{18}$ Our STM measurements show that $1 \mathrm{~T}^{\prime} / 1 \mathrm{~T}^{\prime}$ domain boundaries are wellordered interfaces that exhibit several different structures. By combining scanning tunneling spectroscopy (STS) measurements and first-principles calculations, we have determined that $1 \mathrm{~T}^{\prime} / 1 \mathrm{~T}^{\prime}$ domain boundaries exhibit topologically unprotected one-dimensional (1D) modes that are dispersive near the Fermi level and that exhibit energy dependent decay lengths. These modes reside both inside and outside of the $1 \mathrm{~T}^{\prime}$-bulk bandgap and, unlike $1 \mathrm{~T}^{\prime} / 1 \mathrm{H}$ interface modes, do not directly connect bulk valence and conduction bands.

Results. Mixed-phase single layers of $\mathrm{WSe}_{2}$ were grown using molecular beam epitaxy (MBE) on bilayer graphene (BLG) supported by SiC. These samples exhibit islands that are single domains of either $1 \mathrm{~T}^{\prime}$ or $1 \mathrm{H}$ phase, as well as mixedphase islands with coexisting $1 \mathrm{~T}^{\prime}$ and $1 \mathrm{H}$ domains. ${ }^{2}$ Voltage pulses applied between the STM tip and monolayer $1 \mathrm{~T}^{\prime}-\mathrm{WSe}_{2}$ islands were used to manipulate the $\mathrm{WSe}_{2}$ structural phase. Figure 1 shows STM topographic images of a $1 \mathrm{~T}^{\prime}-\mathrm{WSe}_{2}$ island before and after application of STM tip pulses. The "before" image (Figure 1a) shows a single-domain region of the $1 \mathrm{~T}^{\prime}$ phase with a uniform orientation of atomic rows running from top to bottom (each row contains a zigzag chain of $\mathrm{W}$ atoms $\mathrm{s}^{1,2,9,11}$ ). Figure $1 \mathrm{~b}$ shows the same region after a voltage pulse of $10 \mathrm{~V}$ was applied for $100 \mathrm{~ms}$ between the tip and surface at a constant tip-surface separation of $\sim 6 \AA$. After application of the pulse, the island exhibits multiple domains having different orientations that are connected by ordered $1 \mathrm{D}$ domain boundaries (tip pulses can also cause the formation of adsorbate clusters near domain boundaries, as shown in Figure S7).

Because the $1 \mathrm{~T}^{\prime}$ phase can be formed in three equivalent orientations (via a Peierls distortion of its $C_{3}$-symmetric $1 \mathrm{~T}$ parent phase), several possible $1 \mathrm{~T}^{\prime} / 1 \mathrm{~T}^{\prime}$ domain boundaries are expected. ${ }^{18}$ The most common $1 \mathrm{~T}^{\prime} / 1 \mathrm{~T}^{\prime}$ interface observed in our samples is the $120^{\circ}$ domain boundary, which occurs for $85 \%$ of all observed boundaries and which connects neighboring domains rotated with respect to each other by $120^{\circ}$ (Figure $2 \mathrm{a}$ ). Other observed domain boundaries are the $60^{\circ}$ domain boundary (observed $13 \%$ of the time) and the $0^{\circ}$ domain boundary (observed $2 \%$ of the time), as shown in Figure $2 b$ and $c$ (structural models are shown in Figure $2 d-f$ ). These well-ordered interfaces are straight-line defects that extend up to $20 \mathrm{~nm}$ in length in our samples. The formation of $1 \mathrm{~T}^{\prime} / 1 \mathrm{~T}^{\prime}$ domain boundaries is reversible through the application of a high current raster scan by the STM (300 $\mathrm{mV}, 1 \mathrm{nA}$ ). Such scans remove the adsorbate clusters that form during the generation of $1 \mathrm{~T}^{\prime} / 1 \mathrm{~T}^{\prime}$ domains, likely changing local strain distributions. This provides additional evidence of the ferroelastic nature of $1 \mathrm{~T}^{\prime}-\mathrm{WSe}_{2}$ (further details are discussed in section 6 of the Supporting Information, SI).
The local conversion of single-layer $\mathrm{WSe}_{2}$ from the $1 \mathrm{~T}^{\prime}$ phase to the $1 \mathrm{H}$ phase can be induced using the same voltage pulse method as described above. Figure 1c shows a different single-phase $1 \mathrm{~T}^{\prime}$ island where the dimer chains run from top to bottom before applying a tip pulse, while Figure $1 \mathrm{~d}$ shows the same region after applying a voltage pulse of $10 \mathrm{~V}$ for $100 \mathrm{~ms}$. The tip pulse causes an extended region of the island to convert into a new phase that exhibits reduced apparent height. We identified this region as the $1 \mathrm{H}$ phase of single-layer $\mathrm{WSe}_{2}$ (see Figure S6 for details). Such $1 \mathrm{~T}^{\prime}$ to $1 \mathrm{H}$ phase conversion was only observed in "confined" regions as seen here (i.e., tipinduced $1 \mathrm{H}$ domains were always surrounded by other material).

While $1 \mathrm{~T}^{\prime} / 1 \mathrm{~T}^{\prime}$ domain boundaries could be created by applying tip pulses with $V_{\text {pulse }} \geq 6 \mathrm{~V}$, stronger tip pulses ( $V_{\text {pulse }}$ $\geq 10 \mathrm{~V}$ ) were required to locally induce the $1 \mathrm{~T}^{\prime}$ to $1 \mathrm{H}$ phase transition. These observations agree with predictions that the transition barrier between different orientations of the $1 \mathrm{~T}^{\prime}$ phase should be lower than the barrier for a $1 \mathrm{~T}^{\prime} \rightarrow 1 \mathrm{H}$ transition. ${ }^{18}$ Tip pulses with $V_{\text {pulse }}>6 \mathrm{~V}$ often cause damage to $1 \mathrm{~T}^{\prime}$ islands either by creating holes or by breaking apart the island. However, once $1 \mathrm{~T}^{\prime} / 1 \mathrm{~T}^{\prime}$ and $1 \mathrm{~T}^{\prime} / 1 \mathrm{H}$ domain structures are successfully induced, then they remain stable under normal scan conditions.

To explore the electronic structure of topologically trivial interfaces in a QSHI, we performed STS at the sites of the $120^{\circ}, 60^{\circ}$, and $0^{\circ}$ boundary structures shown in Figure $2 \mathrm{a}-\mathrm{c}$. The $\mathrm{d} I / \mathrm{d} V$ spectra obtained in the $1 \mathrm{~T}^{\prime}$ bulk (green curves in Figure $2 \mathrm{~g}-\mathrm{i}$ ) reflect the $1 \mathrm{~T}^{\prime}-\mathrm{WSe}_{2}$ bulk bandgap, which has an average full width at half-maximum (fwhm) of $85 \pm 21 \mathrm{mV}$ centered at $-130 \pm 5 \mathrm{mV}$ (determination of the bulk bandgap was performed as described in Supplementary Note 3 of ref 2). The spectral weight observed inside the bulk bandgap is explained by lifetime broadening and the $-130 \mathrm{mV}$ offset is due to $\mathrm{n}$-doping induced by the bilayer graphene/SiC substrate, consistent with previous studies. ${ }^{2,20,29}$ The narrow dip at $V=0$ seen in Figure $2 g-i$ likely arises from an interplay between disorder and long-range electron-electron interactions as has been suggested previously. ${ }^{2,5,20,21,27,28}$ This feature is more pronounced at $1 \mathrm{D}$ domain boundaries, which is consistent with predictions regarding disorder-induced behavior in low dimensions. ${ }^{28}$

The spectra for the $120^{\circ}$ and $60^{\circ}$ domain boundaries (Figure $2 \mathrm{~g}, \mathrm{~h}$ ) are similar in that they both have a minimum at $V=0$ and exhibit broad, sloping features in the filled state regime over the range $-300 \mathrm{mV}<V_{\mathrm{S}}<0$. Neither of these domain boundaries shows any significant signatures of the bulk bandgap. The $0^{\circ}$ domain boundary (Figure $2 \mathrm{i}$ ) also has a minimum at $V=0$, but it shows a pronounced dip right in the bulk bandgap energy range. These experimental features are qualitatively different from $\mathrm{d} I / \mathrm{d} V$ spectra observed at topologically protected $1 \mathrm{~T}^{\prime} /$ vacuum and $1 \mathrm{~T}^{\prime} / 1 \mathrm{H}$ boundaries where a clearly defined edge-state peak is seen at the bulk bandgap energy ${ }^{2}$ (a reference $\mathrm{d} I / \mathrm{d} V$ spectrum taken at the topological $1 \mathrm{~T}^{\prime}$ /vacuum edge is shown in Figure S8).

Because the $120^{\circ}$ domain boundaries are the dominant defect feature, we performed a more in-depth study of their spatially dependent electronic structure. Figure 3 shows $\mathrm{d} I / \mathrm{d} V$ maps over the energy range $-400 \mathrm{mV}<V_{\mathrm{s}}<150 \mathrm{mV}$ for a $120^{\circ} 1 \mathrm{~T}^{\prime} / 1 \mathrm{~T}^{\prime}$ domain boundary that intersects a $1 \mathrm{~T}^{\prime} / 1 \mathrm{H}$ domain boundary. The first panel (Figure 3a) shows an STM topograph of the region and includes the $120^{\circ} 1 \mathrm{~T}^{\prime} / 1 \mathrm{~T}^{\prime}$ domain boundary (dashed oval) as well as the $1 \mathrm{~T}^{\prime} / 1 \mathrm{H}$ 
a

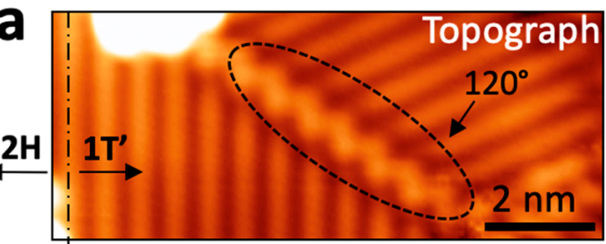

b

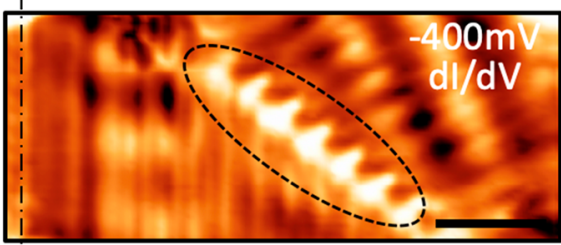

C

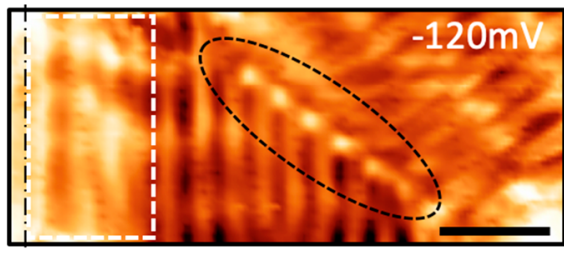

d

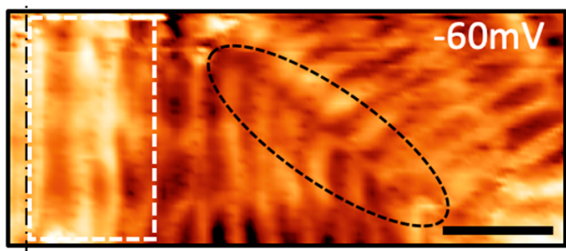

e

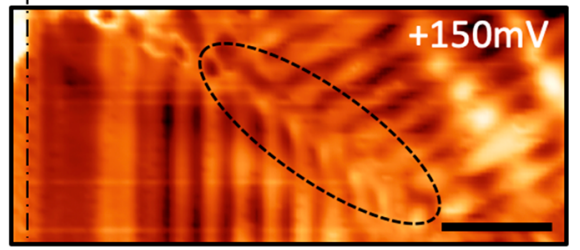

Figure 3. Comparison of electronic properties of $120^{\circ} 1 \mathrm{~T}^{\prime} / 1 \mathrm{~T}^{\prime}$ domain boundary and $1 \mathrm{~T}^{\prime} / 1 \mathrm{H}$ boundary coexisting in single-layer $\mathrm{WSe}_{2}$. (a) STM image of a mixed-phase WSe $e_{2}$ island with a $120^{\circ} 1 \mathrm{~T}^{\prime} /$ $1 \mathrm{~T}^{\prime}$ domain boundary (standard STM topograph). The $1 \mathrm{~T}^{\prime} / 1 \mathrm{H}$ interface is marked by a vertical dashed line, while the $1 \mathrm{~T}^{\prime} / 1 \mathrm{~T}^{\prime}$ interface is outlined by a dashed oval $\left(V_{s}=1 \mathrm{~V}, I_{\mathrm{t}}=10 \mathrm{pA}\right) . \mathrm{d} I / \mathrm{d} V$ maps of the same area are shown for (b) $V_{s}=-400 \mathrm{mV},(\mathrm{c})-120$ $\mathrm{mV},(\mathrm{d})-60 \mathrm{mV}$, and (e) $+150 \mathrm{mV}$. Spectroscopy parameters: $f=$ $613.7 \mathrm{~Hz}, V_{\mathrm{ac}}=4 \mathrm{mV}, I=100 \mathrm{pA}, T=4.5 \mathrm{~K}$. Dashed white box outlines the topologically protected edge-state.

boundary (marked by a vertical dashed line with the $1 \mathrm{~T}^{\prime}$ phase to the right). This area allows us to simultaneously compare the electronic structure of "topological" and "trivial" domain boundaries.

Figure $3 \mathrm{~b}$ shows a $\mathrm{d} I / \mathrm{d} V$ map measured at $-400 \mathrm{mV}$, which corresponds to an energy below the lower edge of the bulk $1 \mathrm{~T}^{\prime}-\mathrm{WSe}_{2}$ bandgap shown in Figure $2 \mathrm{~g}$ (i.e., the bulk valence states). Bright intensity corresponding to high LDOS is observed at the site of the $120^{\circ}$ domain boundary, while the LDOS near the $1 \mathrm{~T}^{\prime} / 1 \mathrm{H}$ interface remains low. Figure $3 \mathrm{c}$ shows a $\mathrm{d} I / \mathrm{d} V$ map at $-120 \mathrm{mV}$, which lies inside the $1 \mathrm{~T}^{\prime}$ bulk bandgap. At this energy, high LDOS intensity is observed at both the $120^{\circ}$ domain boundary and the $1 \mathrm{~T}^{\prime} / 1 \mathrm{H}$ interface region (intensity near the $1 \mathrm{~T}^{\prime} / 1 \mathrm{H}$ boundary originates from the topological edge state). ${ }^{2}$ Figure $3 \mathrm{~d}$ shows a $\mathrm{d} I / \mathrm{d} V$ map measured at $-60 \mathrm{mV}$, which is near the upper edge of the bulk bandgap. Here, high-intensity LDOS is observed near the $1 \mathrm{~T}^{\prime} /$ $1 \mathrm{H}$ interface (from the topological edge state), while the LDOS at the $120^{\circ}$ domain boundary shows low intensity. Figure $3 \mathrm{e}$ shows a $\mathrm{d} I / \mathrm{d} V$ map measured at $+150 \mathrm{mV}$, which corresponds to an energy well into the bulk conduction band. At this energy, neither the topological $1 \mathrm{~T}^{\prime} / 1 \mathrm{H}$ interface state nor the trivial $120^{\circ}$ domain boundary shows high intensity features. The high intensity LDOS localized at the $1 \mathrm{~T}^{\prime} / 1 \mathrm{~T}^{\prime}$ boundary in the $\mathrm{d} I / \mathrm{d} V$ maps indicates the existence of defect states in the energy range $-400 \mathrm{mV}<V_{\mathrm{s}}<-60 \mathrm{mV}$. The broad spectroscopic feature measured in the $\mathrm{d} I / \mathrm{d} V$ point spectrum over this range for $120^{\circ}$ domain boundaries (Figure $2 \mathrm{~g}$ ) can thus be attributed to confined dispersive defect modes. The $120^{\circ}$ domain boundary mode is seen to have a more strongly energy-dependent decay length than the $1 \mathrm{~T}^{\prime} / 1 \mathrm{H}$ interface state and to have more intensity at lower energies.

To clarify the origin of these electronic features, we performed first-principles density functional theory (DFT) simulations. The atomic structure of the interfaces was first relaxed using periodic boundary conditions in a ribbon geometry with a plane-wave basis set ${ }^{22}$ (the relaxed structures are presented in Figure $2 \mathrm{~d}-\mathrm{f}$ ). We then used the nonequilibrium Green's function method (NEGF) to model the line defects with semi-infinite boundary conditions. ${ }^{23,24}$ The resulting spin-dependent electronic band structures for the three different interfaces are presented in Figure $4 a-c$. The number of bands, the dispersion, and the spin character of the defect modes changes dramatically for the different boundary types. Defect modes belonging to the $60^{\circ}$ domain boundary (which has the least amount of symmetry) span the entire bandgap energy region, while the localized states of the $120^{\circ}$ and $0^{\circ}$ domain boundaries do not completely close the bulk energy gap (the bulk bandgap of the $60^{\circ}$ domain boundary model is somewhat affected by the strain that arises from matching bulk lattice constants with the defect periodicity). We see that while the defect modes mostly close the overall energy gap for the $60^{\circ}$ and $120^{\circ}$ domain boundaries, the gap remains bulk-like for the $0^{\circ}$ defect, consistent with our STM spectroscopy observations (Figure $2 \mathrm{~g}-\mathrm{i}$ ). The simulated $120^{\circ}$ domain boundary states are observed to be spin-polarized out of the plane, while for $60^{\circ}$ domain boundaries, the direction of spin polarization rotates for different states within the same band (colors in Figure 4a,b). ${ }^{26}$ The $0^{\circ}$ domain boundaries possess inversion symmetry, and so defect states associated with this boundary show no spin polarization (Figure $4 \mathrm{c}$ ).

Figure $4 d-f$ compare the calculated LDOS of the $120^{\circ}, 60^{\circ}$, and $0^{\circ}$ domain boundaries (blue curves) with the LDOS of the $1 \mathrm{~T}^{\prime}$ bulk (gray curves) as a function of energy (the Fermi level has been shifted to match the experimentally observed ndoping). Overall, we find reasonable agreement between the simulated LDOS in Figure 4 and the corresponding STM spectroscopy measurements of Figure 2. For example, while the experimental bulk bandgap feature vanishes for the $60^{\circ}$ defect (Figure $2 \mathrm{~h}$ ), an energy gap remains for the $0^{\circ}$ defect (Figure $2 \mathrm{i}$ ), similar to the theory plots of Figure $4 \mathrm{e}$ and $\mathrm{f}$. In the $120^{\circ}$ case, the predicted small $10 \mathrm{meV}$ energy gap is likely smeared out by level broadening effects that are observed experimentally but not accounted for in conventional DFT simulations. ${ }^{25}$ When we add Gaussian broadening to our calculation, the $120^{\circ}$ gap feature is smeared out (Figure $4 \mathrm{~d}$ ) similar to what is seen experimentally (Figure $2 \mathrm{~g}$ ). A significant discrepancy between the theory and the data is the pronounced LDOS peak seen near $E=0$ for all three domain boundary types. By contrast, all three domain boundaries show a broad dip at $V=0$ in the STM spectroscopy rather than the predicted peak. This is explained by the coexistence of disorder and electron-electron interactions in these materials, which 

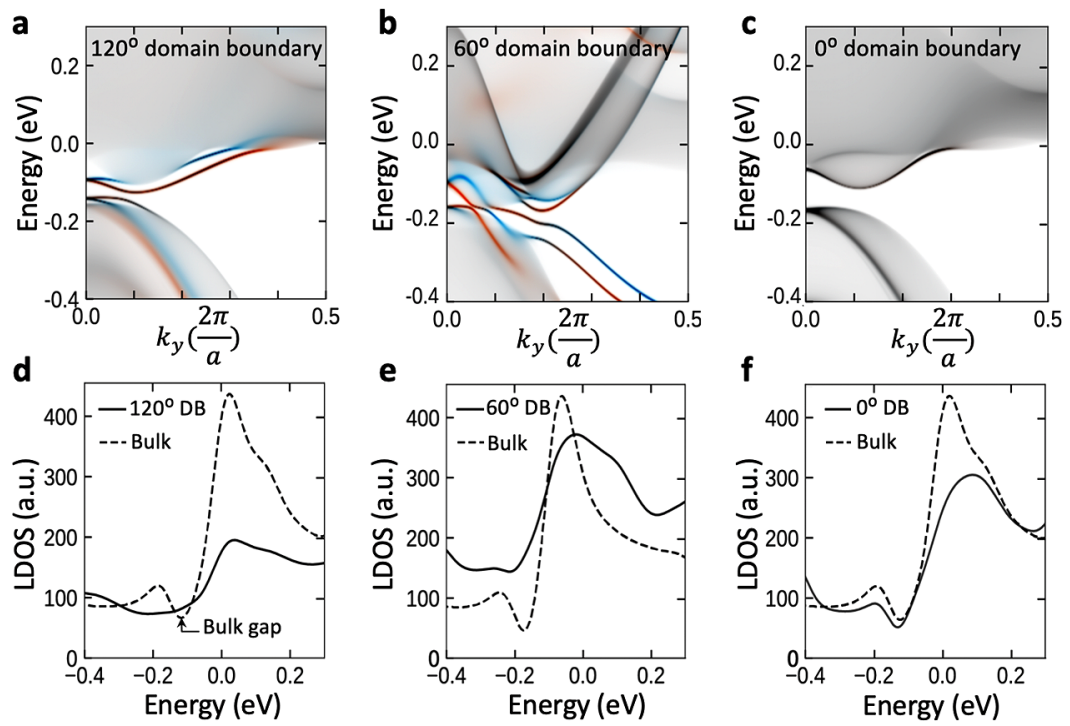

Figure 4. Calculated band structure and local density of states of different $1 \mathrm{~T}^{\prime} / 1 \mathrm{~T}^{\prime}$ domain boundaries. Calculated band structure of $(\mathrm{a}) 120^{\circ},(\mathrm{b})$ $60^{\circ}$, and $(\mathrm{c}) 0^{\circ} 1 \mathrm{~T}^{\prime} / 1 \mathrm{~T}^{\prime}$ domain boundaries for $1 \mathrm{~T}^{\prime}-\mathrm{WS} \mathrm{e}_{2}$ monolayer. Bulk states are gray, and spin-polarized interface modes are red and blue. (d-f) Calculated LDOS of top-layer Se atoms at a domain boundary (black solid curve) compared to LDOS in the bulk (black dashed curve) for (d) $120^{\circ}$, (e) $60^{\circ}$, and (f) $0^{\circ} 1 \mathrm{~T}^{\prime} / 1 \mathrm{~T}^{\prime}$ domain boundaries. The Fermi level $(E=0)$ has been shifted to match experimental data (plots presented in panels $\mathrm{d}-\mathrm{f}$ have been convolved with a Gaussian having $\sigma=30 \mathrm{mV}$ to simulate level broadening effects ${ }^{25}$ ).

open a pseudogap at $V=0 \mathrm{eV}$ but which are not accounted for in DFT simulations.

Conclusion. In conclusion, we have successfully manipulated the local electronic and structural properties of singlelayer $1 \mathrm{~T}^{\prime}-\mathrm{WSe}_{2}$, thereby inducing a local phase transition from the $1 \mathrm{~T}^{\prime}$ to the $1 \mathrm{H}$ phase as well as creating $1 \mathrm{~T}^{\prime} / 1 \mathrm{~T}^{\prime}$ domain boundaries. The induced $1 \mathrm{~T}^{\prime} / 1 \mathrm{~T}^{\prime}$ domain boundaries exhibit different rotational configurations, with a $120^{\circ}$ domain boundary being the most common structure. Our combined STS measurements and first-principles calculations show that these new $1 \mathrm{~T}^{\prime} / 1 \mathrm{~T}^{\prime}$ domain boundaries yield topologically unprotected $1 \mathrm{D}$ states that are dispersive in energy near the Fermi level and exhibit energy-dependent decay lengths. These results create new opportunities for exploring electron- and spin-based devices where charge carriers traveling along QSH edges might be deflected into trivial domain boundary modes in a controllable fashion. ${ }^{12}$

\section{ASSOCIATED CONTENT}

\section{(S) Supporting Information}

The Supporting Information is available free of charge on the ACS Publications website at DOI: 10.1021/acs.nanolett.9b02157.

Detailed position-dependent spectroscopic measurements near a $0^{\circ}$ and $120^{\circ}$ domain boundary, $\mathrm{d} I / \mathrm{d} V$ measurements of the topological edge state, $\mathrm{dI} / \mathrm{dV}$ maps of a $120^{\circ}$ domain boundary, theoretical calculations on the domain boundary structures, electronic structure of $1 \mathrm{~T}^{\prime}$ vacuum termination and LDOS of the three $1 \mathrm{~T}^{\prime} / 1 \mathrm{~T}^{\prime}$ domain boundaries, representative illustration of electronic behaviors (trivial versus nontrivial) of possible interface states, example of trivial/topological two-channel system, experimental evidence for ferroelasticity, experimental detail on tip-induced $1 \mathrm{H}$ domain, theoretical and experimental analysis of the decay length for $120^{\circ}$ domain boundary state (PDF)

\section{AUTHOR INFORMATION}

\section{Corresponding Author}

*E-mail: crommie@berkeley.edu.

ORCID ${ }^{\circ}$

Charlotte Herbig: 0000-0003-3106-1781

Hyejin Ryu: 0000-0003-3600-9755

Michele Pizzochero: 0000-0003-3948-8202

Sung-Kwan Mo: 0000-0003-0711-8514

Michael F. Crommie: 0000-0001-8246-3444

\section{Author Contributions}

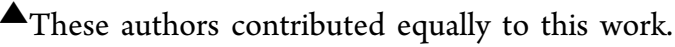

\section{Notes}

The authors declare no competing financial interest.

\section{ACKNOWLEDGMENTS}

This research was supported as part of the Center for Novel Pathways to Quantum Coherence in Materials, an Energy Frontier Research Center funded by the U.S. Department of Energy, Office of Science, Basic Energy Sciences (STM spectroscopy and $\mathrm{d} I / \mathrm{d} V$ mapping). Support was also provided by the National Science Foundation under award EFMA1542741 (surface preparation and topographic characterization). The work performed at the ALS (film characterization) was supported by the Office of Basic Energy Sciences, US DOE under Contract No. DE-AC02-05CH11231. C. H. acknowledges the support of Alexander von Humboldt Foundation for a Feodor Lynen research fellowship. The work performed at the Stanford Institute for Materials and Energy Sciences and Stanford University (MBE growth) was supported by the Division of Materials Science, Office of Basic Energy Sciences, US DOE under contract No. DE-AC0276SF00515. Theoretical modeling of the two-channel conductance by M.P. and E.J.M. was supported by the DOE Office of Basic Energy Sciences under grant DE-FG02-ER45118. M.P. acknowledges support from an NRC Research Associateship award at the U.S. Naval Research Laboratory. S. T. 
acknowledges the support from the CPSF-CAS Joint Foundation for Excellent Postdoctoral Fellows. H. R. acknowledges fellowship support from NRF, Korea through Max Planck Korea/POSTECH Research Initiative No. 2016K1A4A4A01922028. A.P., M.P., and O.V.Y. acknowledge support by the ERC Starting grant "TopoMat" (Grant No. 306504) (ab initio theoretical formalism development), as well as Swiss National Science Foundation grants No. 162612 (2D bulk electronic structure) and No. 172543 (1D interface electronic structure). First-principles calculations were performed at the Swiss National Supercomputing Centre (CSCS) under project s832 and the facilities of Scientific IT and Application Support Center of EPFL. We thank Quansheng Wu for assistance with calculations, and we want to thank Canxun Zhang with helpful discussion.

\section{REFERENCES}

(1) Tang, S.; Zhang, C.; Wong, D.; Pedramrazi, Z.; Tsai, H. Z.; Jia, C.; Moritz, B.; Claassen, M.; Ryu, H.; Kahn.; et al. Quantum Spin Hall State in Monolayer 1T'-WTe2. Nat. Phys. 2017, 13, 683-687.

(2) Ugeda, M. M.; Pulkin, A.; Tang, S.; Ryu, H.; Wu, Q.; Zhang, Y.; Wong, D.; Pedramrazi, Z.; Martín-Recio, A.; Chen, Y.; et al. Observation of Topologically Protected States at Crystalline Phase Boundaries in Single-Layer WSe2. Nat. Commun. 2018, 9, 3401.

(3) Wu, S.; Fatemi, V.; Gibson, Q. D.; Watanabe, K.; Taniguchi, T.; Cava, R. J.; Jarillo-Herrero, P. Observation of the Quantum Spin Hall Effect up to $100 \mathrm{~K}$ in a Monolayer Crystal. Science 2018, 359, 76-79.

(4) Fei, Z.; Palomaki, T.; Wu, S.; Zhao, W.; Cai, X.; Sun, B.; Nguyen, P.; Finney, J.; Xu, X.; Cobden, D. Edge Conduction in Monolayer WTe2. Nat. Phys. 2017, 13, 677-682.

(5) Jia, Z.-Y.; Song, Y.-H.; Li, X.-B.; Ran, K.; Lu, P.; Zheng, H.-J.; Zhu, X.-Y.; Shi, Z.-Q.; Sun, J.; Wen, J.; et al. Direct Visualization of a Two-Dimensional Topological Insulator in the Single-Layer $1 \mathrm{~T}^{\prime}$ WTe2. Phys. Rev. B: Condens. Matter Mater. Phys. 2017, 96, No. $041108(\mathrm{R})$.

(6) Kane, C. L.; Mele, E. J. Quantum Spin Hall Effect in Graphene. Phys. Rev. Lett. 2005, 95, 226801.

(7) Bernevig, B. A.; Zhang, S.-C. Quantum Spin Hall Effect. Phys. Rev. Lett. 2006, 96, 106802.

(8) Hasan, M. Z.; Kane, C. L. Colloquium: Topological Insulators. Rev. Mod. Phys. 2010, 82, 3045.

(9) Qian, X.; Liu, J.; Fu, L.; Li, J. Quantum Spin Hall Effect in TwoDimensional Transition Metal Dichalcogenides. Science 2014, 346, 1344-1347.

(10) Ma, Y.; Kou, L.; Li, X.; Dai, Y.; Smith, S. C.; Heine, T. Quantum Spin Hall Effect and Topological Phase Transition in TwoDimensional Square Transition-Metal Dichalcogenides. Phys. Rev. B: Condens. Matter Mater. Phys. 2015, 92, 085427.

(11) Choe, D.-H.; Sung, H.-J.; Chang, K. J. Understanding Topological Phase Transition in Monolayer Transition Metal Dichalcogenides. Phys. Rev. B: Condens. Matter Mater. Phys. 2016, 93, 125109 .

(12) Phillips, M.; Mele, E. J. Charge and Spin Transport on Graphene Grain Boundaries in a Quantizing Magnetic Field. Phys. Rev. B: Condens. Matter Mater. Phys. 2017, 96, 041403.

(13) Wang, Y.; Xiao, J.; Zhu, H.; Li, Y.; Alsaid, Y.; Fong, K. Y.; Zhou, Y.; Wang, S.; Shi, W.; Wang, Y.; et al. Structural Phase Transition in Monolayer MoTe2 Driven by Electrostatic Doping. Nature 2017, 550, 487-491.

(14) Lin, Y.-C.; Dumcenco, D. O.; Huang, Y.-S.; Suenaga, K. Atomic Mechanism of the Semiconducting-to-Metallic Phase Transition in Single-Layered MoS2. Nat. Nanotechnol. 2014, 9, 391-396.

(15) Keum, D. H.; Cho, S.; Kim, J. H.; Choe, D.-H.; Sung, H.-J.; Kan, M.; Kang, H.; Hwang, J.-Y.; Kim, S. W.; Yang, H.; et al. Bandgap Opening in Few-Layered Monoclinic MoTe2. Nat. Phys. 2015, 11, $482-486$.
(16) Kappera, R.; Voiry, D.; Yalcin, S. E.; Branch, B.; Gupta, G.; Mohite, A. D.; Chhowalla, M. Phase-engineered Low-Resistance Contacts for Ultrathin MoS2 Transistors. Nat. Mater. 2014, 13, $1128-1134$.

(17) Li, Y.; Duerloo, K.-A. N.; Wauson, K.; Reed, E. J. Structural Semiconductor-to-Semimetal Phase Transition in Two-Dimensional Materials Induced by Electrostatic Gating. Nat. Commun. 2016, 7, 10671.

(18) Li, W.; Li, J. Ferroelasticity and Domain Physics in TwoDimensional Transition Metal Dichalcogenide Monolayers. Nat. Commun. 2016, 7, 10843.

(19) Duerloo, K.-A. N.; Li, Y.; Reed, E. J. Structural Phase Transitions in Two-Dimensional Mo- and W-Dichalcogenide Monolayers. Nat. Commun. 2014, 5, 4214.

(20) Song, Y.-H.; Jia, Z.-Y.; Zhang, D.; Zhu, X.-Y.; Shi, Z.-Q.; Wang, H.; Zhu, L.; Yuan, Q.-Q.; Zhang, H.; Xing, D.-Y.; et al. Observation of Coulomb Gap in the Quantum Spin Hall Candidate Single-Layer 1T' WTe2. Nat. Commun. 2018, 9, 4071.

(21) Massey, J. G.; Lee, M. Direct Observation of the Coulomb Correlation Gap in a Nonmetallic Semiconductor, Si: B. Phys. Rev. Lett. 1995, 75, 4266.

(22) Giannozzi, P.; Baroni, S.; Bonini, N.; Calandra, M.; Car, R.; Cavazzoni, C.; Ceresoli, D.; Chiarotti, G. L.; Cococcioni, M.; Dabo, I.; et al. QUANTUM ESPRESSO: a Modular and Open-Source Software Project for Quantum Simulations of Materials. J. Phys.: Condens. Matter 2009, 201, 395502.

(23) Ozaki, T. Variationally Optimized Atomic Orbitals for LargeScale Electronic Structures. Phys. Rev. B: Condens. Matter Mater. Phys. 2003, 67, 155108 .

(24) Ozaki, T.; Kino, H. Numerical Atomic Basis Orbitals from $\mathrm{H}$ to Kr. Phys. Rev. B: Condens. Matter Mater. Phys. 2004, 69, 195113.

(25) Brar, V. W.; Wickenburg, S.; Panlasigui, M.; Park, C.-H.; Wehling, T. O.; Zhang, Y.; Decker, R.; Girit, Ç.; Balatsky, A. V.; Louie, S. G.; et al. Observation of Carrier-Density-Dependent ManyBody Effects in Graphene via Tunneling Spectroscopy. Phys. Rev. Lett. 2010, 104, 036805 .

(26) Bychkov, Y. A.; Rashba, E. I. Properties of a 2D Electron Gas with a Lifted Spectrum Degeneracy. J. Exp. Theor. Phys. Lett. 1984, 39, $78-81$.

(27) Altshuler, B. L.; Aronov, A. G. Zero Bias Anomaly in Tunnel Resistance and Electron-Electron Interaction. Solid State Commun. 1979, 30, 115-117.

(28) Bartosch, L.; Kopietz, P. Zero Bias Anomaly in the Density of States of Low-Dimensional Metals. Eur. Phys. J. B 2002, 28, 29-36. 